\title{
Abbey J. Hardy-Fairbanks, MD
}

\section{Articles:}

Cowman WL, Hansen JM, HardyFairbanks AJ, Stockdale CK. Vaginal misoprostol aids in difficult intrauterine contraceptive removal: a report of three cases. Contraception. 2012 Feb 23. [Epub ahead of print] PubMed PMID: 22364817.

http://dx.doi.org/10.1016/j.contraception. $\underline{2012.01 .007}$ 